\title{
Definiciones y niveles de bullying en una muestra de adolescentes argentinos
}

\author{
Definitions and Levels of Bullying in an Argentinean \\ Adolescent Sample
}

Santiago Resett*

\begin{abstract}
Resumen
Abstract

Introducción: el bullying es un importante factor de riesgo para la salud mental de los adolescentes. Propósito: explorar las definiciones de bullying que brindaban los adolescentes. Muestra: se constituyó una muestra de 202 adolescentes de Paraná, Argentina. Instrumentos: The Cartoons Task de Smith, la pregunta abierta de Vaillancourt y el cuestionario de Olweus. Resultados: sólo un $8 \%$ indicaba el desbalance de fuerzas, un $4 \%$, la repetición y un $10 \%$, la intencionalidad en su definición. En la tarea de Smith, la gran mayoría podía identificar las viñetas que eran bullying, pero también se incluían situaciones que no eran acoso como bullying. Conclusión: las definiciones sobre qué es bullying dada por los adolescentes coinciden solo parcialmente con su definición científica.

Palabras clave: bullying, definición, adolescentes, argentinos

Introduction: bullying is an important risk factor for the mental health of adolescents. Purpose: the present research studied the definitions and level of bullying in adolescents. Sample: a sample constituted of 202 adolescents in Paraná, Argentina. Measures: Smith's Cartoons Task, Vaillancourt's open question, and Olweus questionnaire to measure bullying. Results: only $8 \%$ indicated the imbalance of force, $4 \%$, repetition and $10 \%$, intentionality. In Smith's task, the vast majority could identify bullying vignettes, but they also included situations that were not harassment such as bullying. Conclusion: definitions of what is bullying given by adolescents only partially coincide with the scientific definition of such behavior.

Key words: bullying, definition, adolescents, Argentinean

\footnotetext{
*Consejo Nacional de Investigaciones Científicas y Tecnológicas (CONICET), Buenos Aires, Argentina ; Pontificia Universidad Católica Argentina. Facultad de Psicología y Psicopedagogía, Buenos Aires, Argentina. Mail de contacto: resettsantiago@gmail.com Doi: https://doi.org/10.46553/RPSI.16.32.2020.p7-23
}

Fecha de Recepción: 2 de julio de 2019 Fecha de Aceptación: 19 de mayo de 2020
\end{abstract}




\section{Introducción}

El acoso escolar -bullying en idioma inglés- es considerado un importante factor de riesgo para la salud mental de los niños y adolescentes debido a su asociación con numerosos problemas de ajuste psicosocial (Card, Isaacs \& Hodges, 2007; Nansel, Overpeck, Pilla, Ruan, Simons-Martin \& Scheidt, 2001). No obstante, el acoso puede ser notablemente negativo en la etapa adolescente debido a la mayor conciencia de sí mismo y a la relevancia que cobra el grupo de pares (Paul \& Cillesen, 2003).

Durante mucho tiempo el bullying fue objeto de poco interés por los investigadores. Así en comparación con el maltrato infantil a manos de los padres u otros adultos, el acoso por parte de los pares recién está empezando a ser reconocido como un grave problema de violencia en niños y adolescentes (Runyon, Kenny, Berry, Deblinger \& Brown, 2006).

Olweus $(1993,2013)$ trató de dar una definición precisa sobre qué es acoso escolar. Según este autor, existe acoso cuando un individuo -o grupo de individuos- es expuesto repetidamente a acciones negativas por parte de un sujeto o por parte de un grupo $\mathrm{y}$, generalmente, el sujeto acosado tiene menos fuerza o es más débil que el agresor. Acción negativa se refiere a que el sujeto que acosa tiene la intención o el propósito lastimar o incomodar a otro alumno. Repetidamente implica que el sujeto ha sido expuesto a la agresión frecuentemente. Finalmente, para que el acto se considere bullying, debe observarse una relación asimétrica, una desigualdad en la fuerza física y mental entre ambas personas (Craig, Pepler \& Blais, 2007; Juvonen \& Graham, 2001). Dicha definición ha sido ampliamente aceptada por la comunidad científica (Smith, del Barrio \& Tokunaga, 2012). El bullying puede ser llevado a cabo de distintas formas: verbales -poner apodos, burlarse, insultar-, físicas -golpes, patadas, empujones, morder- y también de un modo relacional o indirecto; esto es, sin usar contacto físico o verbal directo con la víctima: esparcir rumores, dañar la reputación de otro alumno o excluir (Rigby, Smith \& Pepler, 2004). El acoso escolar, consecuentemente, puede entenderse como un subtipo de agresión, pero con características especiales, como la repetición y el desbalance de fuerzas (Nansel et al., 2001; Olweus, 2013). La investigación también demostró que el bullying es un fenómeno social en el que existen roles bien identificados: las víctimas del acoso, los perpetradores, grupos ambos -quienes son victimizados y perpetradores al mismo tiempo- y los espectadores. Estos últimos no son ni víctimas ni perpetradores, pero con sus conductas de inacción y pasividad refuerzan dicha conducta (Juvonen \& Graham, 2001; Olweus, 2013).

Por todo lo dicho, está bien establecida y aceptada la definición de qué es el bullying en la comunidad científica (Smith et al., 2012). No obstante, estudios internacionales han demostrado que cuando los niños y adolescentes son inquiridos sobre qué es para ellos el acoso, rara vez brindan en sus respuestas espontáneas dichas características establecidas por ella. Por ejemplo, sólo un $1,6 \%$ de los adolescentes menciona intencionalidad, 2\%, la repetición y un $26 \%$ señala la desigualdad de poder (Vaillancourt, McDougall, Hymel, Krygsman, Miller, Stiver \& Davis, 2008), aunque un $92 \%$ enfatiza acciones negativas en dicha definición de bullying.

Por este motivo, el Cuestionario de Agresores/Víctimas de Olweus (1996), el 
cual es uno de los más prestigiosos y usados en el primer mundo (Kyriakides, Kaloyirou \& Lindsay, 2006), brinda a los alumnos una definición de acoso con el fin de que los sujetos informen sobre el bullying y no sobre otros tipos de agresiones o conflictos (Phillips \& Cornell, 2012). Sin embargo, dos dificultades se presentan a este respecto: en qué medida los niños o adolescentes tienen en mente dicha definición cuando luego responden si han sido victimizados en las distintas preguntas del cuestionario y que dicha definición impuesta ignora las diferencias culturales entre los distintos países (Smith, Cowie, Olafsson \& Liefooghe, 2002); incluso encontrar en cada lengua y cultura un equivalente al término inglés "bullying" es un desafío considerable. Por ello, en épocas más actuales en los países del primer mundo se han llevado a cabo estudios sobre qué conductas son consideradas acoso en cada cultura y qué términos emplean los niños y adolescentes para referirse al bullying (Smith et al., 2016). Dichas investigaciones han detectado así algunas diferencias relevantes en las distintas naciones a este respeto y en cómo se lleva a cabo. Por ejemplo, en el Japón existe el Ijime: un acoso que llevan a cabo generalmente alumnos de la misma edad de las víctimas, en el aula y perpetrado mediante la exclusión y el ignorar. En cambio, en Inglaterra el bullying se refiere a un tipo de acoso llevado a cabo por alumnos mayores, en el patio y físicamente. Por otra parte, en Italia prepotenza y violenza denotan una acción violenta y más de tipo físico (Smith, Kanetsuna \& Koo, 2007).

De este modo, Smith y colaboradores (Smith et al., 2002; Smith et al., 2016) desarrollaron una serie de Viñetas (Cartoons en inglés) con individuos dibujados con palotes en situaciones que son acoso -como viñetas con conductas no agresivas- para que los niños y adolescentes señalen qué acciones ellos consideran son acoso y por qué. Estudios llevados a cabo en 14 países y 10 lenguas en poblaciones de niños y adolescentes demostraron la dificultad de traducir la palabra bullying en cada una de las lenguas; incluso muchas veces los términos usados en cada región eran coloquiales y no aparecían formalmente en ellas. También destacaron importantes diferencias evolutivas: los niños solamente distinguían situaciones agresivas de no agresivas, mientras que los adolescentes de 14 años ya podían discriminar las peleas del bullying físico, como también identificar el bullying verbal y relacional (Smith et al., 2002).

En la literatura científica, el enfoque del cuestionario de Olweus se enmarcaría en el enfoque Etic. Este enfoque aplica instrumentos estandarizados -generalmente autoinformes-, definiciones o teorías -como la teoría de los Cinco Grandes Factores de la Personalidad (Costa \& McCrae, 1992)desarrollados en las naciones del primer mundo con el fin tratar de replicarlas y validarlas en otras regiones, muchas veces dejando de lado las diferencias culturales, sociales y económicas entre naciones. En cambio, el enfoque Emic o Indogenous -como el de las Viñetas de Smith- busca el significado particular de las conductas y variables en cada una de las distintas regiones, a partir de una exploración desde la subjetividad de los participantes dentro de su propio marco cultural (Cheung, Vijver \& Leong, 2011; Smith et al., 2016). Estudios transculturales demostraron cómo muchos constructos desarrollados en los países del primer mundo no se ajustaban 
adecuadamente en otros contextos culturales (Cheung et al., 2011). De esta manera, uno se puede preguntar, ¿en qué medida la operacionalización del constructo de bullying postulado y comprobado en los países del primer mundo se aplica en los contextos latinoamericanos? Cabe destacar que ambos enfoques son útiles y se necesita de un balance entre ellos para explorar un fenómeno en gran profundidad. Esto es, un balance entre el rigor metodológico del primero y la sensibilidad cultural del segundo (Cheung et al., 2011). La combinación de ambos enfoques en la investigación se conoce como mixto y permite una mayor integración y profundidad del conocimiento (Wisdom \& Creswell, 2013). Ambos tipos de enfoque tienen su utilidad a la hora de describir y entender una conducta de gran relevancia psicosocial como el bullying. El paradigma Emic, con la aplicación del cuestionario de Olweus, por ejemplo, es una herramienta de fácil aplicación y permite evaluar los porcentajes de víctimas y perpetradores en muestras de gran tamaño, como medir otras características importantes del acoso: género, edad, lugares donde ocurre, etc. Al ser el test más usado en el mundo, esto permite la comparación de los resultados con otras investigaciones internacionales. $\mathrm{Su}$ definición de bullying brindada y sus opciones de respuestas temporales a la hora de contestar evitan que se informe sobre otros tipos de conductas conflictivas. En cambio, si se desea conocer el significado del bullying en cada cultura -qué conductas son acoso para los adolescentes- y si dicha definición varía de contexto en contexto, el enfoque Etic es más pertinente, ya que indaga el significado subjetivo de la conducta en el entorno de los participantes. Así, se puede generar conocimiento local sobre qué es el bullying en la Argentina o para el desarrollo de instrumentos locales para su medición. Por otra parte, dicha perspectiva tiene gran implicancia para la prevención del bullying, ya que un primer paso es conocer y entender cómo definen dicho comportamiento los adolescentes. Si se conceptualiza el bullying desde el punto de vista de los adolescentes, luego se puede trabajar en por qué dichas conductas son negativas.

Debido a las importantes diferencias culturales, sociales y económicas de la Argentina y las naciones del primer mundo, sería interesante saber en dicho país cómo los adolescentes definen el bullying, como también observar los niveles de victimización y bullying existentes. Se sabe que la Argentina es un país desarrollado, pero no altamente desarrollado y perteneciente a la tradición latina y católica. A pesar de la relevancia de la presente problemática, en la Argentina pocos estudios se han llevado a cabo para explorar las definiciones de acoso escolar que generan espontáneamente los adolescentes. Asimismo, la pronta identificación de quiénes pueden ser víctimas, agresores y cómo definen los adolescentes el bullying es fundamental para desarrollar medidas preventivas a este respecto. Un primer y gran paso a este respecto sería determinar -desde el enfoque Emic- qué conductas identifican ellos como bullying y por qué, como explorar si dichas definiciones varían según los roles en el bullying -espectador, victimizado y perpetrador-, esto último combinando los enfoques Etic y Emic.

\section{Objetivos}

-Estudiar los niveles de victimización y 
bullying en adolescentes y si varían según el género.

-Explorar las definiciones que brindan del bullying los adolescentes y determinar si los roles en el bullying -espectador, victimizado y perpetrador- introducen diferencias en las definiciones.

\section{Metodología}

\section{Muestra}

Se constituyó una muestra intencional de 202 adolescentes de ambos géneros de entre 12 y 18 años de dos escuelas públicas secundarias de la ciudad de Paraná, Entre Ríos, Argentina. La edad media fue 13.3 $(S D=1.2)$. Un $33 \%$ correspondió a alumnos de primer año, un $29 \%$, a segundo año, un $22 \%$, a tercer año, el $7 \%$, a cuarto y el resto, a quinto año.

\section{Instrumentos}

Cuestionario de Agresores / Víctimas (Olweus, 1996, citado en Olweus, 1993). Es un cuestionario autoadministrado que se completa anónimamente y que consiste en 38 preguntas para medir los problemas de los encuestados con relación a llevar a cabo el bullying/ser victimizado durante los últimos meses. En primer lugar, este instrumento da una definición a los alumnos sobre qué van a entender por bullying, ya que este es un fenómeno complejo y puede ser confundido por ellos con otros tipos de conflictos entre pares (Phillips \& Cornell, 2012).

Algunos ejemplos de preguntas sobre agredir y ser victimizado:

"Desde que empezaron las clases $i$ Te agredieron en estas formas en la escuela?"

"Me pusieron sobrenombres

feos, me hicieron cargadas pesadas, o se burlaron de mí".

Para medir la frecuencia del bullying emplea las siguientes alternativas de respuesta: Nunca, Una o dos veces, Dos o tres veces al mes, Más o menos una vez por semana y Varias veces por semana. Las cuales se puntúan de 0 a 4 . Las virtudes psicométricas del mismo han sido comprobadas en muestras de más de 5.000 sujetos (Olweus, 2013).

Olweus (2013) señala que para considerar que un alumno ha sido victimizado o ha llevado a cabo el bullying debe responder que lo ha sido o la ha llevado a cabo al menos dos o tres veces al mes. En el presente estudio se aplicó la versión que Resett $(2011,2014)$ adaptó a la Argentina con buenas propiedades psicométricas, se emplearon solamente las dos preguntas globales para determinar quiénes fueron victimizados y quiénes agresores. Dicho instrumento está ampliamente validado en la Argentina y su validez concurrente y consistencia interna fueron sólidamente establecidas en muestra de adolescentes de distintas provincias de la Argentina (Resett, 2018).

The Cartoons Task (Smith et al., 2002). El instrumento The Cartoons Task se basa en administrar 40 láminas con situaciones que pueden o no ser acoso escolar. En dichas láminas hay situaciones de agresión física, verbal y relacional con las características del acoso: por ejemplo, un alumno de mayor edad que acosa frecuentemente a otro más pequeño o alguien que es acosado todo el tiempo. Otras que son situaciones negativas que no representan una situación de acoso 
-por ejemplo, un alumno que, sin querer, rompe un útil escolar de otro o insiste para que otro venga a jugar aunque no lo desee-, situaciones de agresión pero que no son acoso -un alumno golpea a otro o una pelea entre dos alumnos- y situaciones de hacer chistes o bromas entre amigos, por ejemplo, Juan y Carlos se hacen burlas amistosas mutuas y se ríen. Finalmente, presenta situaciones de pares que no representan una situación de acoso, sino de cooperación, por ejemplo, un alumno que ayuda a otro. En total se compone de tres láminas de situaciones que no son acoso o bullying -dañar sin querer-, dos de coaccionar a otro para que haga algo, dos de cooperación, dos de hacer bromas amistosas, una de pelea física, tres de agresión reactiva, dos de agresión física, cinco de agresión verbal, diez de agresión relacional, una de cyberagresión, y nueve situaciones de agresión física, verbal y relacional con características de acoso, como desigualdad de fuerzas o reiteración. Las láminas de acoso escolar tratan sobre acoso verbal directo, acoso físico directo y acoso relacional o indirecto. Algunas de ellas enfatizan en la repetición, otras, el desbalance de fuerzas. También se presentan viñetas que hacen hincapié en la discriminación por sexismo o racismo (Smith et al., 2002).

Existen 40 láminas para varones y 40 láminas para mujeres que demuestran situaciones similares con el único cambio de que en una se usan nombres femeninos y en la otra, masculinos. Para aplicarlas en los distintos países de deben usar nombres típicos masculinos y femeninos de la región. Las láminas están hechas con figuras de palotes para evitar cualquier sesgo de etnia o apariencia física. El procedimiento para aplicarlo consiste en evaluar primero en cada país o cultura cómo le dicen al bullying o acoso escolar, preguntando a expertos en el tema, buscando en diccionarios, realizando entrevistas $\mathrm{o}$ grupos focales a niños $\mathrm{o}$ adolescentes, para luego aplicarlo e indagar qué es acoso para los niños o adolescentes. Luego se aplica cada una de las láminas individualmente o en grupos de dos o tres alumnos, se las presenta y pregunta con los términos que se utilizan en cada cultura o nación: ¿esto es.... o no? En el caso de que respondan afirmativamente, los alumnos deben justificar su respuesta de por qué esa conducta es acoso. En la Argentina se llevó a cabo una búsqueda en diccionarios, consultando a investigadores en la temática y docentes de escuelas primaria y secundaria. Se llevó también un estudio piloto con 40 alumnos de escuelas secundarias con algunas de las viñetas como estímulo para explorar cómo se le decía al bullying. Los resultados indicaron que la forma de denominarlo generalmente era "me pelean", "me joden", "me tratan mal, me maltratan" y "me molestan"; sólo tres adolescentes usaron la palabra bullying (Adornetto, 2016; Resett, 2017). Los cuatro términos que emergieron con mayor frecuencia fueron los usados para aplicar las viñetas en el presente estudio. Dicho test se adaptó en estudios exploratorios argentinos con muestras de adolescentes de Entre Ríos y Buenos Aires (Adornetto, 2016; Resett, 2017).

Pregunta libre Agredir, hacer bullying es cuando... (Vaillancourt et al., 2008). En dicha pregunta que consiste en Para mí agredir, hacer bullying es cuando... Los alumnos en media carilla deben escribir qué es para ellos el acoso, con el fin de observar si brindan las tres características principales que tiene el acoso escolar: intencionalidad, 
repetición en el tiempo y desbalance o desigualdad de fuerzas. Esta pregunta fue diseñada y utilizada en el estudio realizado por Vaillancourt et al. (2008). Esta pregunta ya ha sido empleada en la Argentina en estudios preliminares con adolescentes (Adornetto, 2016; Resett, 2017).

Cuestionario demográfico. Se preguntó género, edad, etc.

\section{Procedimiento de recolección de datos}

La participación de los alumnos fue voluntaria y primeramente se mandó una nota en el cuaderno de comunicaciones solicitando el permiso parental. Se explicó, también, que la participación era voluntaria y que las respuestas eran confidenciales y anónimas.

Los cuestionarios fueron administrados en las horas que la escuela destinó a este fin a lo largo del año 2016.

\section{Tratamiento y análisis de datos}

El procedimiento y análisis de datos se realizó a través del Statical Package for
Social Science (SPSS 22), se extrajeron datos estadísticos descriptivos e inferenciales. Las respuestas de las láminas de Smith y la pregunta abierta de Vaillancourt la llevaron a cabo tres estudiantes avanzados de psicología.

\section{Resultados}

En lo relativo a determinar los porcentajes de alumnos víctimas, agresores, ambos -víctimas y agresores- y no involucrados o espectadores, en la tabla 1 se muestran los resultados totales y según género. Los grupos se constituyeron combinando las respuestas a la pregunta global de ser víctimas y de agredir tomando el punto de corte de al menos 2 o 3 veces al mes.

Como se ve en la tabla 1 , se hallaron diferencias marginales según el género $\chi^{2}(3)$ $=6.96 p<.07$ debido a que más varones que mujeres eran agresores y ambos.

Para estudiar las definiciones de bullying que brindaban los adolescentes, esto es el segundo objetivo, se aplicó la pregunta libre Agredir, hacer bullying es cuando... En la tabla 2 se muestran los resultados de las

\section{Tabla 1}

Porcentajes de víctimas, agresores, ambos y no involucrados según género

\begin{tabular}{|c|c|c|c|}
\hline Grupo & & & Total \\
\hline & Varón & Mujer & \\
\hline No involucrados & $81 \%$ & $90 \%$ & $84 \%$ \\
\hline Víctimas & $7 \%$ & $6 \%$ & $7 \%$ \\
\hline Agresores & $8 \%$ & $3 \%$ & $6 \%$ \\
\hline Ambos & $4 \%$ & $1 \%$ & $3 \%$ \\
\hline$N$ & 98 & 104 & 202 \\
\hline
\end{tabular}


respuestas a dicha pregunta.

Como se ve en la tabla 2, un $8 \%$ señaló en su respuesta desbalance de poder, un $4 \%$ repetición y un $10 \%$ intencionalidad. Con respecto a la conducta negativa verbal, un $67 \%$ hizo hincapié en tal característica para dar su definición y un $26 \%$ indicó de que se trataba de una conducta negativa relacional, mientras que un $51 \%$ mencionó una conducta negativa física. Un $53 \%$ destacó una conducta negativa, pero sin especificar qué tipo de conducta. En lo que se refiere a la personalidad de la víctima, un $36 \%$ señaló que hay una característica de la personalidad de él que lo lleva a ser propenso al acoso y un 6\% respondió que quien agrede tiene determinados rasgos de personalidad. En dicha tabla también se muestran los porcentajes de las categorías y ejemplos típicos hallados para cada una de las respuestas.

En lo relativo a las viñetas de Smith et al., en la tabla 3 se muestran las respuestas de los alumnos. Para clasificar las respuestas se preguntó a los alumnos usando los términos que habían emergido en los pasos previos: "me pelean", "me joden", "me tratan mal o me maltratan" y "me molestan" (ver sección de instrumentos).

Al llevar a cabo un análisis de clusters

Tabla 2

Porcentajes de la respuesta a la pregunta "Agredir, hacer bullying es cuando..."

\begin{tabular}{|c|c|c|}
\hline Categoría & Ejemplo & Porcentajes \\
\hline Desbalance de poder & $\begin{array}{l}\text { "Los alumnos que lo hacen se superiores, } \\
\text { mejores, o más que los otros" }\end{array}$ & $8 \%$ \\
\hline Repetición & "Todos los días le dicen cosas feas" & $4 \%$ \\
\hline Intencionalidad & $\begin{array}{l}\text { "Molestas o dejas } \\
\text { en ridículo a alguien queriendo" }\end{array}$ & $10 \%$ \\
\hline Conducta negativa verbal & $\begin{array}{l}\text { "Le decis cosas } \\
\text { feas o apodos que molestan" }\end{array}$ & $67 \%$ \\
\hline $\begin{array}{l}\text { Conducta negativa } \\
\text { relacional }\end{array}$ & $\begin{array}{l}\text { "Lo dejan solo o lo } \\
\text { excluyen" }\end{array}$ & $26 \%$ \\
\hline Conducta negativa física & $\begin{array}{l}\text { "Cuando golpean o } \\
\text { empujan a un chico o chica" }\end{array}$ & $51 \%$ \\
\hline Conducta negativa general & "Molestar a otro alumno" & $53 \%$ \\
\hline Personalidad de la víctima & $\begin{array}{l}\text { "Tiene algún problema mental, o es bajo } \\
\text { o gordo" }\end{array}$ & $36 \%$ \\
\hline Personalidad del agresor & $\begin{array}{l}\text { "Todo agresor se cree más o quiere } \\
\text { resaltar" }\end{array}$ & $6 \%$ \\
\hline
\end{tabular}


jerárquico para observar cómo se agrupaban las respuestas, cinco clusters emergieron: uno de no agresividad, con viñetas que referían a la cooperación, bromas amistosas, romper una cosa sin querer, incomodar o coaccionar (viñetas 9, 10,11, 12, 14, 20, 23 y 24); uno de exclusión o agresión relacional, junto con la situación de cyberagresión y estar en soledad (viñetas 22, 25, 27, 28, $29,30,31,32,33,34,35,36,37,38,39$ y 40); uno de agresión verbal (viñetas 15 , $17,18,19$ y 21); uno agresión física pero sin características de acoso, como peleas, o agresión reactiva (viñetas 1, 2, 4, 6 y 13,) $\mathrm{y}$, finalmente, uno de agresión física, verbal y relacional con características de bullying, como repetición o desbalance de fuerzas (viñetas 3, 5, 7, 8, 16 y 26). Los clusters no variaban según el género ni las edades de los alumnos -comparando las respuestas de los adolescentes menores de 14 años versus los de más de 14 años, ya que la mediana era 13.9 años-.

Como se ve en la tabla 3, la mayoría de los participantes que describían conductas de bullying -repetición o desigualdad de fuerzas- con preguntas con $100 \%$ de respuestas afirmativas, como lo eran las láminas 3, 5, 7, 16, 26 y 37, como también en láminas cercanas al $100 \%$, como la 11 , 27,28 y 38 . Sin embargo, un $100 \%$ indicaba como bullying simplemente a las conductas agresivas, como por ejemplo el empezar a pegar a un alumno -lámina 2-, decir cosas feas a otro -lámina 15- o decir cosas feas a otro por cómo se viste o habla -lámina 17-. También muchos alumnos señalaban como bullying situaciones que no lo eran, como peleas entre alumnos, agresión reactiva o incluso situaciones de juego o broma: láminas $1,4,8$ y 20 , respectivamente.
A continuación, se presentarán como ejemplos algunas de las respuestas dadas por los alumnos para las viñetas que describían conductas con características de bullying como repetición, intencionalidad y desbalance de fuerza.

Para golpear / pegar a alguien más chico -lámina 3-, hacer que un compañero más chico lleve su mochila todos los días -11- y no dejar que un compañero juegue porque va a un grado más chico -lámina 28-, las respuestas enfatizaban que el agresor era más poderoso física o mentalmente:

" $\mathrm{Si}$, es acoso porque al ser más chico no se puede defender" " $\mathrm{Si}$, es bullying porque se está aprovechando de otro más chico"

"Si, porque es una chica más grande contra uno sola"

"Si, porque el más grande se aprovecha y el otro que es más chico y no dice ni hace nada"

También en dichas láminas se señalaban, aunque en menor medida, la intencionalidad:

"Sí, porque lo hacen adrede"

"Sí, porque lo hacen con la intención de lastimarlo"

"Sí, porque lo hacen para que se sienta mal"

En lo relativo a golpear/pegar cada vez que pueden -lámina 5-, decir cosas feas a otro todas las semanas -lámina 16-, no dejar jugar nunca a un compañero -láminas 26- y un grupo de amigos no deja jugar a uno con ellos (lámina 27), las respuestas generalmente destacaban la repetición o frecuencia:

"Si, eso es acoso porque lo hacen muchas veces" 
Tabla 3

Porcentajes a las respuestas del Cartoons Task de Smith et al.

\begin{tabular}{|c|c|c|}
\hline Viñeta & Sí & No \\
\hline 1- Empezar a golpear / pegar el uno al otro (pelea física) & $18 \%$ & $82 \%$ \\
\hline 2- Empezar a golpear / pegar a otro (agresión física) & $100 \%$ & \\
\hline $\begin{array}{l}\text { 3- Empezar a golpear / pegar a alguien más chico (agresión física } \\
\text { con desigualdad) }\end{array}$ & $100 \%$ & \\
\hline $\begin{array}{l}\text { 4- Empezar a golpear / pegar porque otro le dice estúpido/a (agresión } \\
\text { física reactiva) }\end{array}$ & $27 \%$ & $73 \%$ \\
\hline $\begin{array}{l}\text { 5- Empezar a golpear / pegar cada vez que puede (agresión física } \\
\text { reiterada) }\end{array}$ & $100 \%$ & \\
\hline $\begin{array}{l}\text { 6- Empezar a golpear / pegar si el otro no le da su plata (agresión } \\
\text { física reactiva) }\end{array}$ & $100 \%$ & \\
\hline $\begin{array}{l}\text { 7- Empezar a golpear / pegar en grupo (agresión física con } \\
\text { desigualdad) }\end{array}$ & $100 \%$ & \\
\hline $\begin{array}{l}\text { 8- Empezar a golpear / pegar a uno más chico porque este no le hace } \\
\text { caso y lo contradice (agresión física reactiva con desigualdad) }\end{array}$ & $53 \%$ & $47 \%$ \\
\hline 9- Empezar a golpear / pegar en broma y riéndose (broma amistosa) & $4 \%$ & $96 \%$ \\
\hline $\begin{array}{l}\text { 10- Hacer que una compañera lleve su mochila todos los días } \\
\text { (coacción repetitiva) }\end{array}$ & $77 \%$ & $23 \%$ \\
\hline $\begin{array}{l}\text { 11- Hacer que un compañero más chico lleve su mochila todos los } \\
\text { días (coacción repetitiva con desigualdad) }\end{array}$ & $95 \%$ & $5 \%$ \\
\hline 12- Romper un útil sin querer (dañar sin intención) & $2 \%$ & $98 \%$ \\
\hline 13- Agarrar un útil de otro y romperlo (agresión física) & $80 \%$ & $20 \%$ \\
\hline 14- Olvidar un útil y que otro le preste (cooperación) & $1 \%$ & $99 \%$ \\
\hline 15- Decir cosas feas a otro (agresión verbal) & $100 \%$ & \\
\hline $\begin{array}{l}\text { 16- Decir cosas feas a otro todas las semanas (agresión verbal } \\
\text { reiterada) }\end{array}$ & $100 \%$ & \\
\hline $\begin{array}{l}\text { 17- Decir cosas feas por como el otro habla y se viste (agresión } \\
\text { verbal) }\end{array}$ & $100 \%$ & \\
\hline $\begin{array}{l}\text { 18- Decir cosas feas y burlarse porque el otro camina con un bastón } \\
\text { (agresión verbal) }\end{array}$ & $100 \%$ & \\
\hline 19- Decir cosas feas porque el otro es gay (agresión verbal) & $100 \%$ & \\
\hline $\begin{array}{l}\text { 20- Hacer bromas por el pelo del otro y que los dos se rían (broma } \\
\text { amistosa) }\end{array}$ & $40 \%$ & $60 \%$ \\
\hline 21- Cargar por el pelo a otro y que se ponga triste (agresión verbal) & $100 \%$ & \\
\hline
\end{tabular}

Continua en página 17 
Continua en página 16

\begin{tabular}{|c|c|c|}
\hline Viñeta & Sí & No \\
\hline $\begin{array}{l}\text { 22- Enviar mensajes feos con el celular a un compañero } \\
\text { (cyberagresión) }\end{array}$ & $96 \%$ & $4 \%$ \\
\hline 23- Preguntar a un compañero si quiere venir a jugar (cooperación) & $3 \%$ & $97 \%$ \\
\hline $\begin{array}{l}\text { 24- Insistir en que un compañero venga a jugar a pesar de que no } \\
\text { quiera (incomodar) }\end{array}$ & $60 \%$ & $40 \%$ \\
\hline 25- No permitir que un compañero juegue (agresión relacional) & $93 \%$ & $7 \%$ \\
\hline $\begin{array}{l}\text { 26- No dejar jugar nunca a un compañero (agresión relacional } \\
\text { reiterada) }\end{array}$ & $100 \%$ & \\
\hline $\begin{array}{l}\text { 27- Un grupo de amigos no deja jugar a uno con ellos (agresión } \\
\text { relacional con desigualdad) }\end{array}$ & $98 \%$ & $2 \%$ \\
\hline $\begin{array}{l}\text { 28- No dejar que un compañero juegue porque va a un grado más } \\
\text { chico (agresión relacional con desigualdad) }\end{array}$ & $96 \%$ & $4 \%$ \\
\hline $\begin{array}{l}\text { 29- No querer estar con un compañero para una actividad de a dos } \\
\text { (agresión relacional) }\end{array}$ & $92 \%$ & $8 \%$ \\
\hline 30- No tener ningún amigo en la escuela (soledad) & $43 \%$ & $57 \%$ \\
\hline $\begin{array}{l}\text { 31- No dejar saltar a la cuerda porque es del sexo opuesto (agresión } \\
\text { relacional sexista) }\end{array}$ & $96 \%$ & $4 \%$ \\
\hline $\begin{array}{l}\text { 32- No dejar jugar al futbol porque es del sexo opuesto (agresión } \\
\text { relacional sexista) }\end{array}$ & $97 \%$ & $4 \%$ \\
\hline $\begin{array}{l}\text { 33- No hablar con un compañero porque es el favorito de la maestra } \\
\text { (agresión relacional) }\end{array}$ & $100 \%$ & \\
\hline $\begin{array}{l}\text { 34- No querer hablar con un compañero porque este quiere estar con } \\
\text { muchos alumnos y no solo formar parte de un grupo (agresión } \\
\text { relacional) }\end{array}$ & $73 \%$ & $27 \%$ \\
\hline $\begin{array}{l}\text { 35- Dejar de hablar y quedar en silencio cuando un compañero entra } \\
\text { al aula (agresión relacional) }\end{array}$ & $100 \%$ & \\
\hline $\begin{array}{l}\text { 36- Hacer como si un compañero no estuviese allí (agresión } \\
\text { relacional) }\end{array}$ & $100 \%$ & \\
\hline $\begin{array}{l}\text { 37- Pedir a todos que no hablen con tal compañero (agresión } \\
\text { relacional con desigualdad) }\end{array}$ & $100 \%$ & \\
\hline $\begin{array}{l}\text { 38- Pedir a todos que no hablen con un compañero porque es muy } \\
\text { querida por los chicos del sexo opuesto (agresión relacional con } \\
\text { desigualdad) }\end{array}$ & $90 \%$ & $10 \%$ \\
\hline 39- Contar historias feas sobre un compañero (agresión relacional) & $95 \%$ & $5 \%$ \\
\hline $\begin{array}{l}\text { 40- Escribir cosas feas sobre un compañero en las paredes de los } \\
\text { baños de la escuela (agresión relacional) }\end{array}$ & $100 \%$ & \\
\hline
\end{tabular}


"Sí, porque al hacerlo cada vez que pueden el otro se siente mal"

"Sí, porque al hacerlo muchas veces el otro se siente triste y no puede defenderse"

En lo relativo a golpear/pegar en grupo -lámina 7-, pedir a todos que no hablen con tal compañero -lámina 37- y pedir a todos que no hablen con un compañero porque es muy querido por el sexo opuesto -lámina 38-, las respuestas destacaban la superioridad de los agresores o una mayor fuerza física o mental al agredir en grupo:

"Si, porque son cuatro personas contra uno solo"

"Sí, porque al ser muchos, el otro no se puede defender" "Sí, porque al ser muchos, se creen más que los demás"

Finalmente, se llevaron a cabo unas pruebas de $\chi^{2}$ con el fin de determinar si las definiciones de bullying variaban según los roles en el bullying -víctimas, perpetradores, ambos y espectadores-. El grupo de ambos al ser tan mínimo se unificó con el de agresores. No emergieron diferencias en las respuestas a las láminas de Smith et al., aunque sí en la pregunta abierta de Vaillancourt et al. en desbalance de poder y en características de personalidad del agresor. Con respecto al primer atributo, $7 \%$ de los espectadores señalaron dicha característica versus un $24 \%$ de las víctimas, mientras que nadie del grupo agresor la mencionó $\chi^{2}(2)=12.21 p$ $<.01$. En lo referente a las características de la personalidad del agresor, un $8 \%$ de los espectadores señalaron dicho rasgo versus un $33 \%$ de las víctimas, mientras que nuevamente nadie de los agresores la mencionó $\chi^{2}(2)=10.47 p<.01$

\section{Discusión}

El objetivo central de la presente investigación se basaba en observar los niveles de victimización y agresión como las definiciones de acoso escolar o bullying que brindaban los adolescentes. Para este fin se constituyó una muestra de 202 adolescentes de dos escuelas públicas de la ciudad de Paraná, Entre Ríos, Argentina. Los mismos completaron el cuestionario de Olweus para medir los niveles de victimización y agresión, como la pregunta abierta de Vaillancourt et al. sobre qué es agredir o hacer bullying y las viñetas de Smith et al. para explorar las definiciones de bullying que daban. Por ende, la ventaja del presente trabajo fue la de evaluar una temática de gran relevancia psicosocial con técnicas desde el enfoque Etic y Emic.

En relación con el primer objetivo de determinar los niveles de victimización y agresión desde el enfoque Etic, se hallaron $8 \%$ de víctimas, $6 \%$, de agresores y $3 \%$, de ambos, mientras los restantes eran no involucrados 0 espectadores. Dichos porcentajes eran bastantes similares a los detectados en estudios extranjeros. En Suecia los estudios de Olweus arrojaban porcentajes de $9 \%$ y $7 \%$ para víctimas y agresores (Olweus, 1993). Se sabe que el acoso puede variar en su incidencia de acuerdo a la definición operacional realizada. En la Argentina otro estudio -con dicho instrumento de Olweus- halló también un $10 \%$ y $8 \%$ de víctimas y agresores (Resett, 2011). Más varones que mujeres eran agresores y ambos -víctimas y agresores-, lo cual es coincidente con muchos estudios (Nansel et al., 2001; Olweus, 1993).

En lo relativo al segundo objetivo que 
pretendía estudiar las definiciones de acoso escolar que brindan los adolescentes desde el enfoque Emic, se halló que en la pregunta libre de Vaillancourt Para mi agredir, hacer bullying es cuando... un $8 \%$ señaló en su respuesta desbalance de poder, un $4 \%$, repetición y un $10 \%$, intencionalidad. Como se ve, pocos alumnos señalan en su definición las características distintivas del bullying. Con respecto a la conducta negativa verbal, un $67 \%$ la señaló en su definición y un $26 \%$ afirmó que se trataba de una conducta negativa relacional, mientras que un $51 \%$ mencionó una conducta negativa física. Un $53 \%$ señaló una conducta negativa, pero sin especificar qué tipo de conducta. En lo que refiere a la personalidad de la víctima, un $36 \%$ la mencionó y un 6\% informó rasgos de la personalidad del agresor. Esto coincide con los resultados de Vaillancourt et al. (2008), los cuales indicaban que generalmente los niños y adolescentes no incluían los tres criterios establecidos por los investigadores y la comunidad científica para definir al acoso: intencionalidad, repetición y desbalance de poder, aunque muchos hicieron hincapié en comportamientos negativos.

En lo referente a las respuestas sobre el Cartoons Task de Smith et al., las respuestas se podían agrupas en cinco clusters: situaciones de no acoso -como peleas o cooperación-, situaciones agresivas físicas, verbales, relacionales y, finalmente, conductas agresivas físicas, verbales y relaciones con rasgos de bullying, como repetición y desbalance de fuerzas. Sin embargo, en este último clusters la mayoría de las conductas eran de tipo física. Smith et al. (2002) en sus estudios internacionales, en cambio, halló seis clusters: acoso de todo tipo, agresión verbal, agresión relacional, agresión física con rasgos de acoso, agresión física y agresión física-verbal. Que también dicho autor haya obtenido un grupo de respuestas de un acoso principalmente de tipo físico es algo coincidente con la presente investigación. Otro estudio en una muestra turca detectó un patrón algo similar, pero de cuatro clusters: no agresivo o neutral, agresivo físico, verbal y relacional (Ucanok, Smith \& Karasoy, 2011). Sin embargo, el estudio de Smith et al. como el de Ucanok et al. no emplearon las 40 viñetas, por lo cual los resultados no son completamente comparables. Si bien no era objetivo del presente trabajo, los clusters de las respuestas no variaban según el género ni la edad. El género introduce muy pocas diferencias a este respecto, como sugieren los estudios de Smith et al. (2002). Que no se hayan detectado diferencias evolutivas, puede deberse a que se trataba de una muestra de adolescentes, ya que las diferencias en las respuestas se han comprobado al comparar niños y adolescentes (Smith et al., 2002).

Un $100 \%$ de los alumnos decía que sí eran bullying las viñetas que describían conductas de bullying -repetición o desbalance de fuerzas o ambas cosas, entre otras-, como lo eran las láminas 3 (pegar cada vez que se puede a alguien más pequeño), 5 (pegar cada vez que se puede), 7 (golpear en grupo a otro), 16 (decir cosas feas cada vez que se puede), 26 (no dejar jugar nunca a un alumno) y 37 (pedir a todos que no hablen con un alumno). Asimismo, se señalaban en porcentajes cerca del $100 \%$ otras viñetas con conductas de bullying, como hacer que un compañero más chico lleve su mochila todos los días. Generalmente en su explicación de por qué eran acoso señalaban características como repetición, 
intencionalidad o desbalance de fuerzas, por ejemplo, "sí, porque lo hacen todo el tiempo", "sí, porque lo hacen adrede" o "sí, es bullying porque se está aprovechando de otro más chico", respectivamente. Esto concuerda con los estudios de Smith et al. (2002), los cuales señalaron que los adolescentes pueden distinguir e identificar el bullying. Sin embargo, todos los alumnos $(100 \%)$ señalaron que acoso también era simplemente pegar, golpear -lámina 2-; golpear, pegar a otro para que me dé la plata -lámina 6-; decir cosas feas a otro -lámina 15- o burlarse de alguien porque camina con bastón -lámina 18-. En sus respuestas señalaban que era acoso por ser una agresión intencional o sin provocación de la víctima, aunque no tuviera los rasgos de repetición y desbalance de fuerzas del bullying. Del mismo, muchos alumnos señalaban como bullying situaciones que no lo eran, como peleas entre alumnos, agresión reactiva o incluso situaciones no agresivas, como juegos violentos entre alumnos o cargarse amistosamente. De este modo, las definiciones de acoso que poseen los adolescentes son más amplias y diferentes que las aceptadas por la comunidad científica, ya que, si bien identifican la reiteración y la desigualdad como características del acoso, también incluyen conductas agresivas que no son bullying o situaciones que ni siquiera son agresión. Estos resultados concuerdan con los estudios internacionales en distintos países y lenguas de Smith et al. (2002, 2012, 2016). Así también los hallazgos eran similares a los de las preguntas abiertas de Vaillancourt et al. (2008), en donde una amplia mayoría señalaba una conducta negativa -pegar o insultar- como bullying.

Interesantemente, al observar si los roles en el bullying introducían diferencias en las definiciones, se halló que las víctimas y los espectadores señalaban en mayor medida el desbalance de poder y la personalidad del agresor en su definición, en comparación con los perpetradores del acoso y el grupo ambos. Esto indicaría que quienes perpetran el acoso, según los datos del cuestionario de Olweus, podrían tener una mayor dificultad para conceptualizar el acoso cómo un abuso de poder. Dicha dificultad-tal vez- explicaría por qué llevan a cabo mayores conductas agresivas. Esto coincide con numerosos estudios que señalan que los perpetradores son sujetos insensibles, con poca empatía y una visión positiva de la agresión (Olweus, 1993, 2013). Que solamente en dichos dos atributos hayan emergido diferencias según el rol del bullying -medido con el enfoque Etic- sugiere nuevamente que hay poca coincidencia en la definición científica del bullying y en cómo entienden subjetivamente los adolescentes esta conducta. No obstante, el empleo de ambos enfoques (Etic y Emic) permite un conocimiento más completo y profundo de una conducta como el bullying, poniendo de manifiesto que solo de forma parcial hay coincidencia en cómo definen dicha conducta los adultos y los adolescentes. En lo relativo a las limitaciones de la presente investigación, se empleó el autoinforme como recolección de datos, el cual tiene reconocidas limitaciones -principalmente en un tema como el acoso, como deseabilidad social o la falta de honestidad de las respuestas-. Por otra parte, la muestra era pequeña y no probabilística, por lo cual no es posible hacer una generalización de los resultados obtenidos. Asimismo, se trataba de un estudio de corte transversal, por lo cual no hay un 
seguimiento del fenómeno a lo largo del tiempo. Si bien el Cartoons de Smith et al. y la pregunta abierta de Vaillancourt et al. son técnicas ampliamente usadas en el mundo, se debería explorar cómo funcionan en muestras de adolescentes de otras regiones de la Argentina, ya que su aplicación en la Argentina de ha limitado a muestras de Entre Ríos y Buenos Aires. Asimismo, el combinar técnicas de recolección de datos cualitativas y cuantitativas es una fortaleza, pero un enfoque mixto no está exento de dificultades. El cuestionario de Olweus brinda una definición de qué es bullying, por lo cual en sus respuestas quedan excluidas las conductas que para los adolescentes son acoso desde su punto de vista.

En lo referente a recomendaciones para futuros estudios, se recomienda ampliar la muestra y que sea seleccionada en forma aleatoria. Sería deseable realizar comparaciones transculturales con otros países de la región. Se recomienda, asimismo, realizar un estudio longitudinal para poder explorar cómo van cambiando los roles de víctimas o agresores, como también observar si a lo largo de la adolescencia y, sobre todo, en los adolescentes de mayor edad las definiciones de bullying son más complejas o no. También sería adecuado explorar las definiciones de acoso en la niñez. Por otra parte, sería interesante indagar las definiciones que los adultos brindan sobre el bullying, por ejemplo, docentes o padres. Además de utilizar el autoinforme, sería deseable utilizar nominaciones de grupo de pares o docentes para evitar los sesgos del autoinforme. Del mismo modo, se recomienda que futuras investigaciones sigan empleando la combinación de los enfoques
Etic y Emic para el estudio del bullying y que lo hagan de una forma más profunda, ya que en el presente estudio simplemente se usaron dos preguntas del cuestionario de Olweus, las cuales se relacionaron con las definiciones del acoso. En la presente investigación se le dio más énfasis al enfoque Emic porque se deseaba explorar las definiciones de acoso.

Finalmente, a nivel de las instituciones educativas se recomienda también para la prevención del bullying el uso de los enfoques Etic y Emic, aunque evaluando cuándo debe ser empleado uno u otro. Por ejemplo, en una primera instancia se puede emplear el Emic para explorar qué es para los adolescentes el acoso. Luego que se ha definido y conceptualizado qué es el bullying y se ha trabajado en su prevención, el cuestionario de Olweus puede ser una herramienta adecuada y de rápida implementación para medir si los porcentajes de bullying han disminuido o no. También el enfoque Emic puede incorporarse cuando los programas de intervención para disminuir el acoso tienen escasos resultados: ¿dicha conducta es caracterizada de forma diferente por los niños o adultos? o ¿el programa de intervención es visto negativamente los alumnos y docentes? Del mismo modo, se puede usar el cuestionario de Olweus para identificar a los adolescentes con altos niveles de conductas agresivas y usar el enfoque Emic para tratar de entender por qué llevan a cabo dichas conductas. Asimismo, el seguir profundizando las definiciones de bullying a nivel local puede permitir a futuro el desarrollo de instrumentos estandarizados, como el cuestionario de Olweus, para medir dicha conducta en la Argentina. 


\section{Referencias}

Adornetto, J. (2016). Acoso escolar: definiciones de acoso escolar en adolescentes (Tesis de grado). Universidad Argentina de la empresa, Buenos Aires, Argentina.

Card, N. A., Isaacs, J., \& Hodges, E. V. (2007). Correlates of school victimization: Recommendations for prevention and intervention. En J. E. Zins, M. J. Elias, \& C. A. Maher (Eds.), Bullying, victimization, and peer harassment: A handbook of prevention and intervention. Nueva York: Haworth Press.

Cheung, F. M., van de Vijver, F. J. R., \& Leong, F. T. L. (2011). Toward a New Approach to the Study of Personality in Culture. American Psychologist, 66(7), 593-603 doi: 10.1037/a0022389

Costa, P. T., Jr., \& McCrae, R. R. (1992). Revised NEO Personality Inventory (NEO-PI-R) and NEO Five-Factor Inventory (NEO-FFI) professional manual. Odessa, FL: Psychological Assessment Resources.

Craig, W., Pepler, D., \& Blais, J. (2007). Responding to bullying what works? School Psychology International, 28(4), 465-477.

Juvonen, J., \& Graham, S. (2001). Peer harassment in school. The plight of the vulnerable and victimized. Nueva York: Guilford Press.

Kyriakides, L., Kaloyirou, C., \& Lindsay, G. (2006). An analysis of the Revised Olweus Bully/Victim Questionnaire using the Rasch measurement model. British Journal of
Educational Psychology, 76(4), 781-801.

Nansel, T., Overpeck, M., Pilla, R., Ruan,. W., Simons-Martin, B., \& Scheidt, P. (2001). Bullying behavior among U.S. youth: prevalence and association with psychosocial adjustment. Journal of the American Medical Association, 285(16). 2094-2100.

Olweus, D. (1993). Bullying at school: What we know and what we can do. Cambridge, MA: Blackwell.

Olweus D. (1996). The Revised Olweus Bully/Victim Questionnaire. Bergen, Noruega: HEMIL, Universidad de Bergen.

Olweus, D. (2013). School bullying: Development and some important challenges. Annual Review of Clinical Psychology, 9, 751-780.

Paul, J., \& Cillessen, A. (2003). Dynamics of Peer Victimization in Early Adolescence Results from a FourYear Longitudinal Study. Journal of Applied School Psychology, 19(2), 25-43.

Phillips, V., \& Cornell, D. (2012). Identifying victims of bullying: Use of counselor interviews to confirm peer nominations. Professional School Counseling, 15, 123-131.

Resett, S. (2011). Aplicación del cuestionario de agresores/víctimas de Olweus a una muestra de adolescentes argentinos. Revista de Psicología de la UCA, 13(7), 27-44.

Resett, S. (2014). Bullying: víctimas, agresores, víctimas-agresores y 
correlatos psicológicos. Actas Psiquiátricas y Psicológicas de la América Latina, 60(3), 171-183.

Resett, S. (2017, agosto). Definiciones del bullying en adolescentes. Trabajo presentado en el VIII Congreso Internacional de Educación, Santa Fe, Argentina.

Resett, S. (2018). Análisis psicométrico del Cuestionario de Agresores/ Víctimas de Olweus en español. Revista de Psicología de la PUCP, 36(2), 575-602.

Rigby, K., Smith, P., \& Pepler, D. (2004). Working to prevent school bullying: Key issues. En P. K. Smith, D. Pepler, \& K. Rigby (Eds.), Bullying in schools: How successful can interventions be? (pp. 112) Cambridge: Cambridge University Press.

Runyon, M., Kenny, M., Berry, E., Deblinger, E., \& Brown, E. (2006). Etiología $\mathrm{y}$ vigilancia en el maltrato infantil. En J. Lutzker (Ed.), Prevención de violencia. Investigación $y$ estrategias de intervención basadas en la evidencia (pp. 21-44). México: Manual Moderno.

Smith, P., Cowie, H., Olafsson, R., \& Liefooghe, A. (2002). Definitions of bullying: A comparison of terms used, and age and sex differences, in a 14-country international comparison. Child Development, 73, 1119-1133.

Smith, P., del Barrio, C., \& Tokunaga, R. (2012) Definitions of bullying and cyberbullying: How useful are the terms? En S. Bauman, J. Walker, \& D. Cross (Eds.), Principles of cyberbullying research. definitions, measures and methodology (pp. 26-40). Nueva York y Londres: Routledge.

Smith, P. K., Kanetsuna, T., \& Koo, H (2007). Cross-national comparison of 'bullying' and related Terms: Western and Eastern perspectives. Contemporary research on aggression: School violence. World Meeting of the International Society for Research on Aggression, 3, 3-9.

Smith, P. K., Kwak, K., Hanif, R., Kanetsuna, T., Mahdavi, J., Lin, S., Olafsson, \& Ucanok, Z. (2016). Linguistic issues in studying bullying-related phenomena: Data from a revised cartoon task. En P. K. Smith, K. Kwak, \& Y. Toda (Eds.), School Bullying in Different Cultures: Eastern and Western Perspectives (pp- 280-298). Cambridge: Cambridge University Press.

Ucanok, Z., Smith, P. K., \& Karasoy, D. (2011). Definitions of bullying: Age and sex differences in a Turkish sample. Asian Journal of Social Psychology, 14, 75-83. doi: 10.1111/j.1467-839X.2010.01334.x

Vaillancourt, T., McDougall, P., Hymel, S., Krygsman, A., Miller, J., Stiver, K., \& Davis, C. (2008). Bullying: Are researchers and children/youth talking about the same thing? International Journal of Behavioral Development, 32(6), 486-495.

Wisdom, J., \& Creswell, J.W. (2013). Mixed Methods: Integrating Quantitative and Qualitative Data Collection. Rockville, MD: Agency for Healthcare Research and Quality. 